routine cardiography in patients with uncomplicated leptospirosis are specifically due to that disease. Indeed, it seems probable that they are the non-specific result of a febrile infection. However, previous reports make it clear that pericarditis, endocarditis, and arrhythmias also occur in leptospirosis. These conditions are not seen in artificially induced fever, and cannot be accounted for in this way. Their pathogenesis is unknown, but they could be due to spontaneous bleeding in the myocardium or pericardium.

Spontaneous bleeding often occurs in leptospirosis. Nearly half the patients in this series bled from the lungs, the intestine, the skin, the nose, beneath the conjunctiva, or into the cerebrospinal fluid. A fifth had microscopic haematuria. This bleeding could be very severe indeed. Thus one patient who subsequently recovered from a gross generalized haemorrhagic tendency showed a fall of haemoglobin from $100 \%$ to $47 \%$ (packed cell volume 19\%) in seven days. A necropsy on a similar fatal case seen elsewhere in Malaya showed that the lungs, pleura, gastro-intestinal tract, retroperitoneal spaces, kidneys, heart, and pericardium were riddled with easily visible haemorrhages of varying sizes. Haemorrhage of this severity is uncommon, but milder internal bleeding must often occur. It is not necessary to surmise such an event to explain the E.C.G. changes observed in this series, but the occurrence of such a spontaneous bleed into the pericardium or into the mvocardium near the conducting tissue could, as suggested by Hume and Szekely (1944), account for the cardiac abnormalities not attributed to exudates, infiltrates, sympathetic overactivity, and fever.

\section{Summary}

Serial electrocardiographic studies were done on 25 patients with leptospirosis and 18 patients with malaria who had no evidence of cardiovascular disease on routine clinical examination.

Identical abnormalities were found in the majority of records in the two groups.

After comparison with findings reported in artificial hyperpyrexia, it is concluded that the abnormalities are the nonspecific result of a febrile infection.

A possible cause of arrhythmias and pericarditis in leptospirosis is discussed.

I wish to thank Dr. Paul Szekely for his help and advice with this study.

\section{REFERENCES}

Dawson, B., and Hume, W. E. (1916-17). Quart. F. Med., 10, 90.

Hume, W. E., and Szekely, P. (1944). Brit. Heart \%., 6, 135 .

Knies, P. T., (1941). Amer. Heart f., 22, 804.

Lnieschkin and Wilkins, Baltimore.

Mackay-Dick, J., and Robinson, J. F. (1957). f. roy. Army med. Cps, $103,186$.

Sodeman, W. A., and Killough, J. H. (1951). Amer. F. trop. Med., 31, 479.

\title{
Peripheral Sensorimotor Neuropathy Associated with a Localized Myeloma
}

\author{
D. I. RUSHTON,* M:B., CH.B.
}

Brit. med.7., 1965, 2, 203-205

Neurological manifestations in diseases of the reticuloendothelial system are not uncommon and are said to occur in $15-50 \%$ of cases (Aita, 1962). The vast majority of these cases show neurological symptoms as a result of direct invasion or compression of the nervous tissues. However, in a small number of cases neurological manifestations are severe and yet there is no direct involvement of the nervous system. These may be similar to those syndromes associated with carcinoma.

Victor at al. (1958) presented five cases of sensorimotor neuropathy associated with myeloma, in three of which post-mortem examination revealed no evidence of invasion of the nervous system. Barron et al. (1960) described three cases in which local infiltrations of the peripheral nerves by plasma cells led to demyelination of the adjacent nerve fibres. Other cases of neuropathy associated with myeloma have been reported by Hassan and Yousef (1959) and Boudin et al. (1961), and the condition has been noted by Osserman (1959).

The case reported below is believed to be a case of the type described by Victor et al. (1958).

\section{Case History}

A man aged 45 first noticed coldness and numbness of his feet in January 1962. This was dismissed as being due to the cold weather. However, the condition progressed and paraesthesiae of his lower limbs developed below the knees. In July he had marked difficulty in walking and experienced numbness of his hands.

In August he was admitted to the Midland Nerve Hospital for investigation. At this time he had great difficulty in walking, paraesthesiae of the hands and feet, and increasing clumsiness when using his hands.
Clinical examination revealed extensive neurological manifestations in an otherwise normal male. These were: The upper limbs showed loss of power, areflexia, impaired pinprick and vibration sense, and loss of touch sensation below the elbows. The lower limbs showed gross loss of power, areflexia, absent joint sense, and loss of pinprick and vibration sense below the costal margin. The plantar response was absent. These findings were symmetrical.

After admission his condition slowly progressed, and while in hospital he developed deep-vein thrombosis of his calf; this led to pulmonary embolus, from which he made a satisfactory recovery with the aid of anticoagulant therapy.

On 6 September a bony lesion of D 11 was first noted on an $x$-ray film ; it was thought to be myeloma, but no other confirmation of this diagnosis was obtained.

The neuropathy was provisionally diagnosed as a form of GuillainBarré syndrome, and steroid therapy was begun. This led to a slight temporary improvement in the power of his legs; but it was not maintained, and the symptoms and progression of the disease continued.

In May 1963 he was transferred to Selly Oak Hospital. At this time he had paraesthesiae of all limbs, intermittent dysarthria, areflexia, gross impairment of sensation, and severe attacks of pain in the lower limbs. Muscular weakness was severe. The fundi showed blurring of the disks. On exercise he became very dyspnoeic, probably as a result of involvement of his respiratory muscles. Gradually over the next few months his condition deteriorated and he developed gangrene of the right fifth toe. By August severe sensorimotor neuropathy of his upper limbs, shoulders, and lower limbs was evident. The extremities were cyanosed and the gangrene of the right foot was progressing.

* Department of Pathology, Selly Oak Hospital, Birmingham. Now Research Fellow, Department of Morbid Anatomy, Children's Hospital, Birmingham. 
Chest $x$-ray examination at this time revealed a right hilar shadow, possibly carcinoma of the bronchus. Chest infection ensued and the patient died on 1 September 1963.

\section{Clinical Investigations}

While he was in hospital extensive investigations were carried out, and these are enumerated below.

The cerebrospinal fluid was not examined in the last 11 months of life. Two C.S.F. examinations in the earlier phase of the illness revealed a protein level of $240 \mathrm{mg} . / 100 \mathrm{ml}$. and on one occasion a white-cell count of $5 / \mathrm{c} . \mathrm{mm}$. The manometrics were normal. The sugar was slightly elevated at $88 \mathrm{mg} . / 100 \mathrm{ml}$. The remaining constituents were normal. Serum proteins: two estimations revealed albumin levels of 3.9 and $3.7 \mathrm{~g} . / 100 \mathrm{ml}$., with globulin levels of 3.0 and $3.1 \mathrm{~g} . / 100 \mathrm{ml}$. respectively. Electrophoresis showed a raised alpha-2 fraction. Immuno-electrophoresis revealed a high concentration of gamma- and macrogamma-globulin consistent with an immunological reaction rather than with myeloma. Glucosetolerance curves carried out on two occasions within three weeks of each other revealed a mild diabetic state, the blood sugar at one hour being $250 \mathrm{mg} . / 100 \mathrm{ml}$. in the first curve. Random blood sugars varied between 71 and $130 \mathrm{mg} . / 100 \mathrm{ml} . \quad X$-ray examination revealed no relevant changes in the chest. Examination of the skull, spine, proximal femora, and pelvis revealed a single mixed lytic and sclerotic lesion of D 11 . Cone views showed a sparse trabecular area, chiefly in the right half of the body and right pedicle, associated with an area of sclerosis. Muscle biopsy revealed the appearances of a progressive sensorimotor neuropathy without evidence of a severe loss of nerve fibres.

Repeated haemoglobin estimations varied between 16.0 and 11.6 g./100 ml., with a level of $13.5 \mathrm{~g}$. immediately before death. The E.S.R. (Westergren) rose slowly from 7 to $31 \mathrm{~mm}$. in one hour during the illness. Sternal marrow was normal (9 October 1962). Gum biopsy was negative for amyloid. Repeated testing of the urine did not reveal Bence Jones protein. No autoimmune antibodies were detected, muscle and nervous tissue being included in the antigens tested. Other investigations revealed a positive thymol flocculation test, serum cold agglutinins at $4^{\circ} \mathrm{C}$. positive $1: 5$ and $1: 10$, and a positive viral complement-fixation test for respiratory virus.

The following investigations were normal: serum alkaline and acid phosphatases, bilirubin, thymol turbidity, complement, electrolytes and urea, urinary 5-hydroxyindoleacetic acid, porphyrins, and porphobilinogen. Barium meal and follow-through, pyruvatetolerance test, blood and C.S.F. Wassermann reaction (and Kahn on the former). The resting calf-blood-flow of the right leg was normal on 31 August 1962.

\section{Post-mortem Examination}

Necropsy was carried out 36 hours after death. The body was that of a rather thin but adequately nourished male with a moderate degree of muscle-wasting of the limbs, no particular groups being more affected than others. The right foot showed early dry gangrene.

The oral cavity, pharynx, larynx, thyroid, and cervical lymph nodes appeared normal. The trachea and bronchi contained a large quantity of mucopus, and the mucosal surfaces were inflamed. The left lung was firmly adherent to the chest wall and numerous loculated cysts were present in the pleura. These contained strawcoloured fluid. Both bases showed some degree of collapse and patchy bronchopneumonia. The upper lobes were emphysematous and the apices contained healed tuberculous foci. The hilar glands were normal in size and appearance. The diaphragm and pericardium were congested, with finely granular surfaces. The heart (330 g.) was of normal size. The myocardium was pale, and small areas of fibrosis were present. Both coronary arteries were atheromatous, the right artery being more severely affected in that it was almost occluded immediately beyond the origin. The valves and endocardium appeared normal. The salivary glands, oesophagus, stomach, duodenum, and small intestine were within normal limits. The colon was packed with hard faeces. The rectum was normal.

The liver $(2,600$ g.) was pale, with a slightly greasy cut surface. The spleen $(450$ g.) was enlarged and the cut surface showed prominent Malpighian bodies. The pancreas, gall-bladder, and bile- ducts were normal. The adrenals were small, with narrow yellow cortices. The kidneys (left $185 \mathrm{~g}$., right $170 \mathrm{~g}$.) were of normal size, with smooth subcapsular surfaces and pale flabby parenchymata. Mild pyelitis, ureteritis, and cystitis were present. The prostate was not enlarged. The aorta was minimally atheromatous, but the arteries of the lower limbs were more severely affected. The right femoral artery was partially occluded by thrombus and the left popliteal artery completely occluded. Both femoral veins contained ante-mortem thrombus.

The limb muscles were pale, fibrous, and atrophied. The abdominal muscles were very pale and thin. The vertebral and sternal marrow were normal apart from that of D 11, which was invaded by a white translucent mass adherent to but not penetrating the spinal dura. The underlying cord was not compressed. The only other tumour deposit was local invasion of the paravertebral tissues adjacent to the involved vertebra.

The cerebrum, cerebellum, pons, and mid-brain were all macroscopically normal. The skull meninges and vessels of the central nervous system seemed to be normal. There was no macroscopical lesion of the spinal cord.

\section{Histological Findings}

Kidney.-The majority of the glomeruli were normal though a few hyalinized tufts were present. Small foci of round-cell infiltration were scattered throughout the sections examined. A few foci of acute inflammatory cells were present, involving small groups of cortical tubules. In some areas these amounted to small abscesses, and the associated tubules held granular casts containing cell debris. The renal arterioles showed some intimal proliferation, but these changes were minimal.

Sections of kidney, spleen, liver, peripheral nerve, and muscle showed no evidence of amyloid deposits.

The remaining significant findings were confined to the nervous and muscular systems and to the tumour.

$D$ 11. - The marrow cavity was almost entirely filled with a typical myelomatous deposit containing plasma cells, often bizarre in shape, with occasional binucleate cells. Similar cells were found in the tissues adjacent to the vertebrae, though the spinal dura was not penetrated. The body trabeculae of the vertebra showed gross thickening in one area of the section, with obliteration of marrow cavity. Sternal and other vertebral marrow sections showed no significant increase in plasma cells.

\section{Nervous System}

Tissues were stained by haematoxylin and eosin, Luxol fast blue (for myelin), Glees and Marsland (for axons), and oil red $\mathrm{O}$ on frozen sections. Examination of the cerebrum, cerebellum, and pons revealed no significant abnormality.

Spinal Cord.-(a) Cervico-medullary junction: The posterior columns showed positive staining with oil red $\mathrm{O}$ and pallor on staining for myelin. The anterior horn cells appeared normal. (b) Cervical cord features were similar to those of the cervicomedullary junction, except that a few anterior horn cells showed central chromatolysis. (c) Thoracic cord (T 1): The anterior horn cells showed central chromatolysis to a greater extent than the cervical cord. The anterior nerve roots showed demyelination and positive oil red $\mathrm{O}$ staining. (d) Sacral and lumbar cord (L 4-5, S 2-3): The changes were similar to those in the thoracic cord, though much more extensive, with the majority of the anterior horn cells showing chromatolysis and shrinkage. Some showed accumulation of brownish pigment in the cytoplasm. The anterior nerve roots showed extensive demyelination, with similar though lesser changes in the posterior nerve roots.

The posterior columns showed demyelination and positive oil red O material.

\section{Peripheral Nerves}

Sciatic Nerves.-There was extreme demyelination. A few fibres showed fragmented myelin. The axons showed irregular outlines with fusiform swellings. There was a slight reduction in numbers. Both nerves were involved equally. Similar changes were present in the sacral roots, though to a less extent than in the peripheral 
nerves. A few of the neural arterioles showed perivascular accumulations of lymphocytes. The posterior root ganglia from similar levels showed loss of distinction between cell components, chromatolysis, and accumulation of brown pigment in the cytoplasm of the neurones. Many neurones appeared as uniform granular areas with indistinct outlines. Irregular patchy demyelination of a lesser degree than that found in the anterior roots was present. The axons appeared normal in numbers, though there was some irregularity in shape.

Thoracic Roots, Median Nerve.-The anterior roots showed demyelination with some irregularity of the axons. These changes were less marked than those in the lumbar region. The median nerve showed more pronounced changes than the nerve roots. The posterior root ganglia showed minimal changes, with early demyelination.

\section{Muscle}

Right Vastus Internus.-The muscle fibres were atrophied and showed loss of striation. The fibrous elements were increased. Many clumps of chromatin-rich material made up of small groups of nuclei were present. Most of the nuclei were peripheral to the muscle fibres, but a few appeared to lie within the cytoplasm of the cells. A few muscle fibres showed vacuolation. In the section examined no normal fibres were found. The general appearance was that of an atrophied but hypercellular muscle. There was no significant cellular infiltration.

Rectus Abdominis.-Changes similar to those found in the vastus muscle was present, though they were less uniform in that bundles of normal fibres were seen among the atrophied fibres.

Sternomastoid.-The histology was essentially normal.

\section{Discussion}

Neurological manifestations in myelomatosis may be the result of compression of the nervous system as with extradural deposits or vertebral collapse. Deposits may directly invade the peripheral nerves or these may be secondarily involved as a result of amyloidosis occurring with the myeloma.

In these cases anatomical and physiological mechanisms explain the symptoms and signs. However, in a small group of cases there is no evidence of direct involvement of the nervous system, yet there are severe and progressive symptoms and signs of neurological dysfunction.

The principal difficulty in these cases is to determine if the two conditions are related. This at present cannot be proved one way or the other, but the similarity to the carcinomatous neuropathies suggests that there may be a direct relationship. Another major problem is to exclude direct involvement of the nervous system, but the extent of the neurological lesions and the absence of myeloma cells make it unlikely that the nervous system is directly involved. In this respect the three cases described by Barron et al. (1960) are of interest as they show a similar though not identical clinical picture and direct involvement of the nervous system. The foci of plasma cells in the nerves were associated with local demyelination. Mild diabetes was present in the case presented, but the clinical picture was not that of diabetic neuropathy. The diabetes was not thought to be important in the aetiology of the neuropathy in this case.
The clinicopathological syndrome appears quite constant in these cases. There is a relentless progressive sensorimotor neuropathy associated with degeneration of the anterior horn cells, nerve roots, and peripheral nerves that is more severe peripherally. Associated with this there is degeneration of neurones in the posterior root ganglia and demyelination of the posterior columns of the cord, especially the columns of Goll. This dying back of the sensory and motor neurones has been described by Gray et al. (1955) in carcinomatous sensory neuropathy and also by Coers and Woolf (1959). The changes suggest a peripheral lesion and the possibility of the primary changes occurring in muscle or muscle nerve-endings.

The aetiology of this syndrome remains obscure. Several possibilities have been considered. The tumour may produce some toxic metabolite which acts specifically on muscle or nerve.

Immunological mechanisms have been suggested, and Wilkinson (1964) has shown antibodies in serum to central nervous tissue and dorsal root ganglia, but not to peripheral nerve, in four cases of carcinomatous sensory neuropathy. Antibodies were not detected in other forms of carcinomatous neuropathy. It was observed in this case that there was lymphatic infiltration, and perivascular lymphocytic cuffing was present in the peripheral nerves though not in the dorsal root ganglia. No antibodies to nervous or muscular tissues were detected.

Serum immunoelectrophoresis in the present case suggested an immunological reaction, but this cannot be directly related to the neurological lesions with the information available. The need for careful search for malignancy in cases of obscure neuropathy is underlined by this case. It is likely that in many cases the tumour will not be found before necropsy is performed.

\section{Summary}

A case of peripheral sensorimotor neuropathy is presented which was associated with myeloma. Few other cases of this association have been reported in the literature. Post-mortem examination failed to reveal any direct invasion of the nervous system by the tumour.

Possible aetiological factors are mentioned and the need to search for malignancy in obscure neuropathies is stressed.

I would like to thank Dr. R. D. T. Cape for permission to publish the clinical findings in this case, and Dr. J. S. McKinnell for his help and guidance in the presentation of the pathological material.

\section{REFERENCES}

Aita, J. A. (1962). Neb. St. med. f., 47, 142.

Barron, K. D., Rowland, L. P., and Zimmerman, H. M. (1960). F. nerv. ment. Dis., 131, 10.

Boudin, G., Pepin, B., and Brion, S. (1961). Bull. Soc. med. Hôp. Paris, 77, 490.

Coers, C., and Woolf, A. L. (1959). Innervation of Muscle. Blackwell, Oxford.

Gray, K. W., Woolf, A. L., and Wright, E. A. (1955). Guy's Hosp. Rep., 104, 157.

Hassan, A. H., and Yousef, M. M. (1959). 7. Egypt. med. Ass., 42, 386.

Osserman, E. F. (1959). New Engl. F. Med., 261, 1006

Victor, M., Banker, B. Q., and Adams, R. D. (1958). F. Neurol. Neurosurg. Psychiat., 21. 73.

Wilkinson, P. C. (1964). Lancet, 1, 1301. 